TP Periodica Polytechnica

Transportation Engineering

45(3), pp. 168-174, 2017

https://doi.org/10.3311/PPtr. 10708

Creative Commons Attribution (i)

RESEARCH ARTICLE

\section{Technical Specification Methodology for an Automotive Proving Ground Dedicated to Connected and Automated Vehicles}

\author{
Zsolt Szalay $^{1}$, Ádám Nyerges ${ }^{1 *}$, Zoltán Hamar ${ }^{2}$, Mátyás Hesz ${ }^{1}$
}

Received 06 March 2017; accepted 18 March 2017

\begin{abstract}
Today's vehicles already have several driver assistant systems and in the near future highly automated vehicles will also appear in road transport. Higher automation levels rely on disruptive technologies that cannot be tested and approved in the former way. To be able to guarantee future road safety also disruptive testing and validation methods are required. The complexity of the systems and the stochasticity of the potential traffic situations demand new approaches with different testing levels and approval layers. Since there are no off-the-self solutions available beyond the research the authors also participate in international activities like the Gear 2030 EU level initiative. This paper will discuss the proposed new approach for connected and automated vehicle testing methodology concluding with the technical specification results for the new Hungarian automotive proving ground.
\end{abstract}

\section{Keywords}

Connected and Automated Vehicles, Autonomous Driving, Self-driving Vehicles, testing and validation, test track, proving ground, Gear 2030

\section{Introduction}

Thanks to the recent revolution of science and technology, vehicles have more and more automated features or systems. Initially the main motivation was to make driving easier or more comfortable, but world megatrends have oriented the development towards to lower fuel consumption, higher traffic safety and reduced environmental impact.

To reach these future objectives it is necessary to increase the level of automation of road vehicles. At the end of the day, automated vehicles are going to appear in everyday transportation. Automated vehicles will overcome today's cars in efficiency, comfort, safety, velocity and traffic density. Connected cars have another advantage: with intelligent traffic control systems, traffic jams can be decreased or even avoided.

Driving a road vehicle is a very complex controlling task, so substituting the human driver with a computer is a real challenge also from the technical side. Due to the new components and increased in-vehicle system complexity, vehicle testing and validation became different as earlier. Testing the vehicle, the driver-controller and the traffic situations together requires new testing methods and strategies. The aim is the same as earlier: to guarantee road safety with reliable operation of the systems. This proof needs well-prepared test and certification procedures. Mistakes should be handled with zero tolerance because it is easy to miss the trust of the society in automated technologies.

There are strong efforts on an EU level towards harmonising the European vision on connected and automated vehicles for the following decades like the Gear 2030 initiative that technical, legislation and financing challenges.

When talking about the motivation of automated driving one of the biggest expectation is the radical reduction of the accident number and severity, since $94 \%$ of the current traffic accidents can be traced back to the human drivers (Tettamanti et al., 2016).

The penetration of automated vehicles will definitely be gradual, so there will always be "conventional vehicles" around. But we can have an estimate of the future potential in automated vehicle business based on Clifford Winston's 
calculations, where he said that the rolling-out of the automated vehicles will result in an economic profit around 200 billion USD per year in the US (Winston, 2016).

This paper is created by the framework of RECAR program, which is a project to facilitate the development of connected and automated vehicles. The aim is to cooperate with the automotive organisation units (companies, research centres, educational institutes and national authorities), learning by doing researches and to bring the new knowledge into education.

Instead of connected and automated vehicles there are also a few expressions like autonomous vehicles and self-driving cars. We use the phrase "connected and automated vehicles" as we feel it the most accurate.

\section{Vehicle testing and validation}

To analyse and compare the testing possibilities of conventional and autonomous road vehicles, the control loop is a good choice. In a conventional vehicle the controller is the human driver. In vehicles with higher SAE levels (SAE International, 2014), the autonomous controller has bigger tasks in vehicle control. The different control loops present the layers between the systems. During vehicle testing these layers and systems has to be tested, and they define the requirements for a laboratory or for a test track.

The purpose of the testing process can be classified by different aspects:

- Component or system operability or cooperability.

- Reliability.

- Durability.

- Security (cyber security).

- Tests in deterministic or stochastic situations.

- Data managing, privacy protection.

- Insurance and liability aspects.

\section{1 "Conventional" vehicle testing and validation}

A conventional vehicle has three main subsystems: the human driver, the vehicle in the environment and the interface between them. Vehicle manufacturers typically test the behaviour of the vehicle in various environments and traffic situations. Test cases are usually focused on the dynamic properties of vehicles. The target is defined by the requirements of the users for example better handling.

The interface between the human driver and the vehicle has two directions. The first is the perception of the vehicle's behaviour. It has three ways: visual, acoustic and haptic. The other direction is the human driver's actuation in the movement of the vehicle. In these layers the better handling is also an advantage.

Testing the driving skills in conventional vehicles has not got big emphasis. It is only important for diving licenses and in other special cases.

Vehicles with driver assistant systems have more layers and systems, these elements need new test methods. The appearance of these systems made necessary the adaptation of software test methods in the automotive industry.

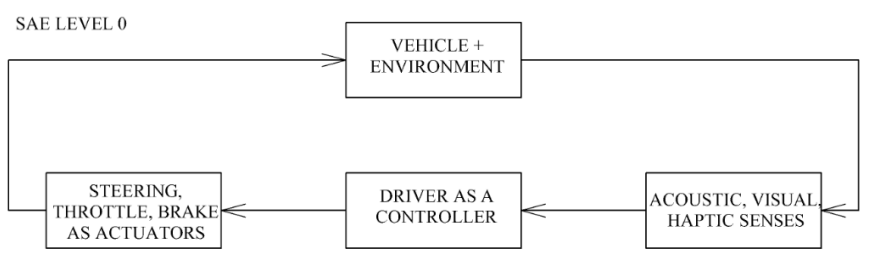

Fig. 1 Control loop of a conventional vehicle

\subsection{Connected and automated vehicle testing and validation}

The vehicle control task which earlier has been done by the human driver is very complex, but it also has a big development potential.

The appearance of connected and automated vehicles changes the testing methods. An SAE level 5 automated vehicle does not need a human driver at all, he/she becomes an additional passenger. Due to this change, the handling of the vehicle will not be as much important as earlier.

As it was mentioned in above, an important motivation of the development of connected and automated vehicles is to achieve better safety in road transportation. The tests focus on the reliability of the autonomous controller which is partially an active safety system in this case. Testing the driving assistant systems or the autonomous driver is similar to test a human driver in a conventional vehicle. But proving that a computer is more reliable than a human driver is a difficult task and it generates moral questions too.

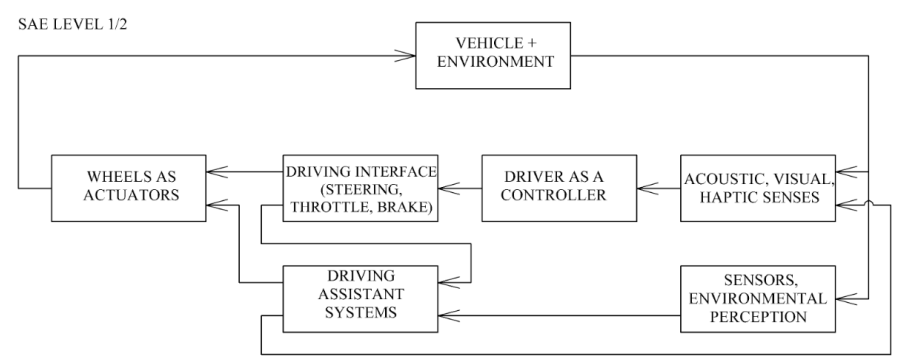

Fig. 2 Control loop of a driver assisted vehicle

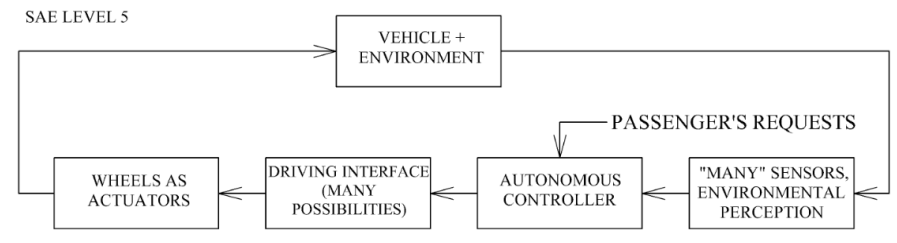

Fig. 3 Control loop of an automated vehicle

The control loops can also help to present the components and the interfaces which should be tested. This can be seen on Fig. 2 with driving assistant systems and on Fig. 3 about a fully automated vehicle. 
The components and interfaces:

- Vehicle and the environment: this is the area of the conventional vehicle tests.

- Environmental perception: the sensors of the vehicle which observe or measure the vehicle dynamics, the trajectory of the vehicle, the road, the other road users, the traffic, the objects around the vehicle and other environmental conditions.

- Controllers of driver assistant or autonomous systems: these components are software (with their hardware units).

- Actuators, which drive the vehicle.

- C2X communication systems in connected vehicles.

The trajectory of the vehicle influenced or controlled by software which can communicate to other vehicles through different network systems. This architecture reveals new risks in vehicles like software bugs, data privacy or cyber security. To cover these risks vehicles require fail safe systems and protected communication networks. Testing and validation procedures should prove the safety of all these components.

\section{Challenges in connected and automated vehicle testing and validation}

Concerning the challenges of connected and automated vehicle testing one has to mention also the necessity of the periodical technical inspections (PTI), but we state that in this paper we focus on the testing requirements of the type approval.

There are various challenges for testing connected and automated vehicles (CAVs) depending on the use-cases, functions and selected automation levels (SAE levels). As a consequence, the testing and validation tasks should also be organised in a similar manner. The challenges not only reflect the vehicles itself but since CAVs are part of the traffic infrastructure, it is still unclear now how to design, develop and deploy the corresponding testing infrastructure for higher levels of automated driving. Furthermore, a competitive proving ground has to have the capabilities not just to test, but certify and validate automated driving functions in a reproducible and efficient way. It is still a question where are the limits of the OEM self certification and where should there be an independent organization come into the picture. It is important to mention that an independent organization cannot certify a function without having worked together with the developers from an early phase.

Another aspect of testing is that almost everybody is aware of the importance of vehicle cyber security, but there is still a lot to do in determining how to secure connected vehicles from manipulation and prevent misuse to guarantee safety. The impact of digitalised mobility solutions, to maximise user experience in the car and the connected vehicles also represent a potential threat for cyber attacks.
In automotive development the optimal utilization of the resources has high importance. In component or system testing using just the necessary resources is a financial requirement. Therefore there are different testing levels are required by connected and automated vehicles:

- Simulation.

- Laboratory.

- Test track/proving ground.

- Limited/controlled public road.

- Public road.

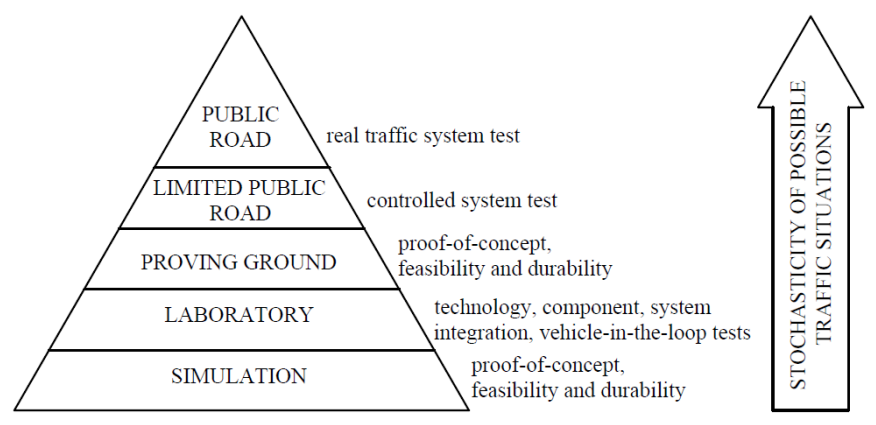

Fig. 4 AD Vehicle Testing \& Validation Pyramid (Szalay, 2016)

The testing of connected and automated vehicles typically focus on the traffic situations. There are studies where the traffic situations are classified to simplify the tests, but the typical property of traffic situations is the stochasticity. It is impossible to pre-define every traffic situation, and it is impossible to prove the reliability of connected and automated vehicles without public road tests. The purpose is to test the systems step by step from the simulation environment to the public road. Step by step testing results in a cost and time efficient demonstration of the reliable operation of connected and automated vehicles.

The testing levels have connections with the V-model of system's development. Simulation tests focus on feasibility. Laboratory tests allow to build the vehicle from components to the whole system. Open road tests can prove the safety.

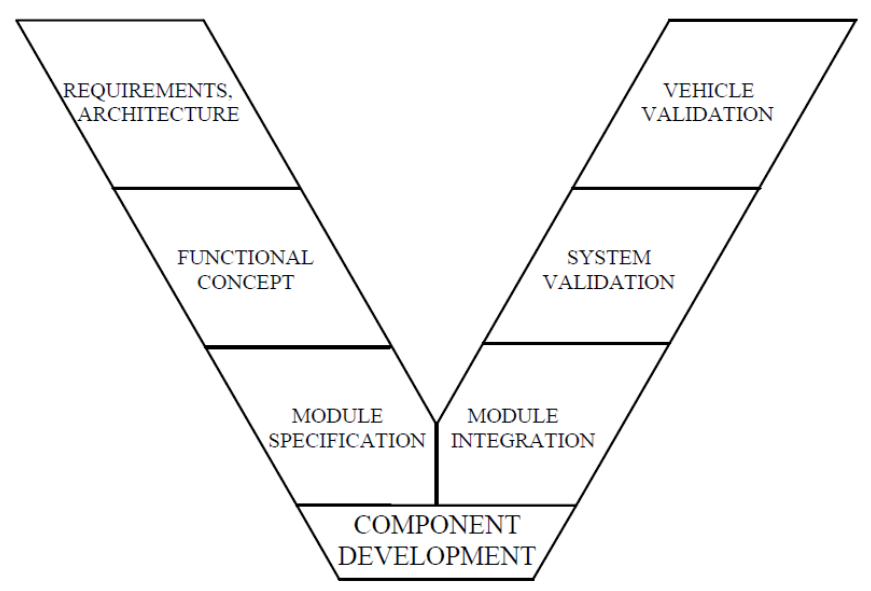

Fig. 5 The V-model of system development 
As considered above, in connected and automated vehicle testing the aim is to prove that the vehicle will make right deliberate decisions in various traffic situations. Due to the uncountable cases of traffic situations, public road tests became more important than ever before. Only public road tests can offer enough stochasticity of various traffic situations.

A well-defined and structured architecture of testing and validation methods could be a basis of a certification process in the future to prove the safety operation of connected and automated vehicles.

In the next few paragraph the levels of the "pyramid" will be discussed.

\subsection{Simulation}

Simulation is the mathematical modelling of a well-defined part of the real world. To reach evaluable results by simulation it is important to define the required complexity of the models. Knowledge of capability, the neglected effects and the limits of the simulation model make the design process efficient.

The next step is to use the available simulation software for modelling road vehicles and traffic situations. From a functional point of view we consider that model-in-the-loop (MIL) and software-in-the-loop (SIL) tests are carried out within this layer.

There are two types of commercial automotive simulation software. The first type focuses on the vehicle dynamics: the integrated vehicle dynamics simulation software. In conventional vehicles the important requirements were safety and easy handling. Designing a car according to these expectations requires the simulation of the vehicle dynamics to analyse the system's operation: engine performance, brake performance, suspension performance and their harmonisation. These simulation environments implement the longitudinal, the lateral and the vertical vehicle dynamic equations. The role of the stochasticity is small here. Some typical examples of this software are CarSim, CarMarker, VI-CarRealTime, Dyna4 Driver Assistance, Dymola, EB Assist ADTF and dSpace Motiondesk. Most of these simulation environments can handle basic traffic situations too (they can test the present driver assistant systems).

The second type focuses on traffic situations. These simulation environments have been developed for transport engineers, to design the traffic flow of cities. In this software the vehicle models are very simple. The aim is to make the transport in complex traffic networks faster. Some examples for this software are PTV VISSIM, CityTrafficSimulator, MovSim and UAF.

The simulation environment which represents the future for automated vehicles integrates these two key features, where the vehicle is handled by the part of the environment, not an independent system. A typical example for this is PTV VISSIM which is able to simulate automated vehicles in every SAE level. In the near future many simulation environments will be developed accordingly.
Another aim of traffic situations is to simulate the interaction between the various road users. For example implementing the vulnerable road users (VRU) prepares the possibility of their perception and to simulate the $\mathrm{C} 2 \mathrm{X}$ communication. There are researches on the protection of the pedestrians and the cyclists by vehicle sensors, typically based on video cameras. The shape and the movement of VRUs are distinctive, and with this information their position can be predicted (Wisch et al., 2016; Large et al., 2016; Bartels et al., 2016).

During the simulation of the different sensors of automated vehicles it is important to analyse the different operating conditions which affect the reliability of the environment perception. Every type of sensor has weaknesses, for example the weather condition has a strong effect on the visual capability of the video based sensors. From another aspect, disturbances can also be simulated by region dependent road types or traffic signs too.

\subsection{Laboratory tests}

The operation of components and systems can be analysed in laboratory tests. Apart from the operation, it is also important to test reliability, durability and cyber security. Laboratory tests lead to much more relevant information of these properties than simulations.

In our approach laboratory types for dedicated automotive testing can be categorised as follows:

- Technology research laboratory, where the basic research is carried out. The enhancement of existing and the development of new systems for CAVs, it is important to test the basic operation principals, for example the wave propagation for RADAR technology. The technology in this sense can be classified as sensors, signal processing and actuators. These laboratories required only general equipment. New communication technologies, like Galileo or $5 \mathrm{G}$, make this type of laboratory very important today.

- Component analysis laboratory, where a vehicle specific function can be tested. An example is a laboratory station, where the video based environment perception can be analysed. In this type of laboratory the same products from different manufacturers can be analysed and compared, and it is possible to do benchmark analyse. The challenge is to simulate the missing environmental conditions. This can be performed by real equipment or by digital signals. This type of laboratory is also called HIL (hardware-in-the-loop) labs.

- System integration laboratory, where the cooperation can be tested between different systems. It is the half-way between the HIL and the VIL (vehicle-in-the-loop) tests. The complexity is higher than a single component test lab and it generally requires complex network systems of the laboratory station (Aradi et al., 2014). 
- Vehicle-in-the-loop (VIL) laboratory, where the complete vehicle is tested without driving it. The test system is rather complex and it requires quite a lot of space, especially when an automated truck is tested. The biggest challenge is to simulate the environment around the vehicle. This can be done by projected videos or by moveable objects, depending on the vehicle's tested environmental perception technology. The aim is to have as many simulated signals as possible, thus leaving the vehicle under test intact. VIL tests are necessary when the safety risk of the test is high, and the vehicle is not prepared enough for open road tests.

\subsection{Proving ground tests}

The main advantage of test tracks is the predictable safety, since they are not publicly accessible. As considered above, there are many systems that requiring different test equipment. A universal test track can serve all expectations.

The typical requirements for a proving ground will be presented below in the specification of the new Hungarian proving ground in Zalaegerszeg a few paragraphs below.

\subsection{Limited public road tests}

There is no doubt that automated vehicles must also be tested on public roads before getting a homologation (type approval). We have the feeling that between the quite well controlled proving ground tests and the fully open public road testing there has to be an intermediate testing and validation layer. This layer would be a controlled area of a city, where the traffic regulations are modified to guarantee safety. This controlled area would be a dedicated "Test City". It might also be incorporate in a so called "smart city"- we would like to use the synergy effect with a Smart City environment. Modified regulation could be an exclusion of certain vehicle categories, typically the vulnerable traffic participants: pedestrians, cyclists, motorcycles, etc.

In summary, this controlled area can serve two aims:

- Testing the automated vehicles, using the controlled stochasticity of the partially public road. The modified regulation of the traffic could be time dependent and it would be dynamically changeable to always reach the safety objectives.

- Apart from the tested self-driving functions of the vehicles, the controlled area could be a "Smart City" too. "Smart City" would be a place, where connected car features and smart traffic control systems could be tested among the conventional traffic stakeholders.

\subsection{Public road tests}

Like many other countries, Hungary also searches the ways to enable public road testing of automated vehicles under certain circumstances. Within the framework of the RECAR coordination office the Hungarian proposal was formulated involving authorities and industrial partners. The proposal is under public consultation now and is expected to be released officially within months. After long discussions, the Hungarian approach shifted to the self-certification direction putting all the liability to the highest ranking officer of the vehicle developer company.

As it was told earlier public road tests are necessary for connected and automated vehicles too, because only these tests can reach enough stochasticity of traffic situations. The development of conventional vehicles also needs public road tests, but there are not as many risks as with automated vehicles.

Due to safety risks the main criterion of the public road tests is the legal environment changes. The working group made recommendations for regulatory changes. The major points of the proposed changes are:

- Definition of the levels of vehicle automation and definition of automated test vehicle.

- Criterions to allow an automated and connected vehicle to public roads (vehicle parameters and notification of tests).

- Conditions to allow a human driver to drive an automated and connected vehicle on public road.

- Designating the roads which are allowed to be used by automated and connected vehicles (see Fig. 6) (Szalay, 2016).

- Specifying the recorded data requirements.

- Exactly defining the liability.

\section{Automotive proving ground concept in Hungary}

In the beginning of 2016 the Hungarian government decided to build an automotive proving ground primarily for automated and connected vehicles. The aim of the investment is to make the Hungarian automotive industry more competitive, which is a major industrial sector of the country. The chosen place is near Zalaegerszeg, the size of the area is 250 ha.

The proving ground should be suitable for a wide range of vehicle and traffic tests for conventional and connected and automated vehicles. Test processes can be performed for certifications, to control regulations, for $\mathrm{R} \& \mathrm{D}$ and for educational aims.

Within the RECAR coordination office the corresponding preparatory tasks have been delegated to dedicated working groups to coordinate the requirements of the different organisation units involved. The tasks were allocated in a way that the requirements represented by the automotive industry. These participants were delegated to the proving ground technical specification working group. There is also an info-communication and digitisation (data and communication) working group.

\subsection{Participants}

The first step was to organise the working group dedicated to the specification of the proving ground. The aim was to invite every relevant stakeholder from the Hungarian automotive industry. 
The whole process was coordinated by Budapest University of Technology and Economics as the leading higher education institute in Hungary.

The participants classified by their sphere in the automotive sector:

- Industrial companies: AImotive, AVL, Bosch, Continental Corporation, Evopro, Knorr Bremse, National Instruments, ThyssenKrupp Presta, TÜV Rheinland, ZF Hungária Kft.

- Research centres and universities: Budapest University of Technology and Economics, MTA SZTAKI,

- National authorities and governmental bodies: EMMI, NFM, NGM, NKH.

Under strict NDA conditions the official meeting started in March 2016 and specification of the proving ground was finished by the end of November in 2016. The specification proposal was handed over to the project company responsible for the proving ground.

\subsection{Proving ground specification}

Without compromising the NDA agreement we can only summarize the conceptual findings during the specification work to enlighten the methodology that we used to collect the requirements.

According to the initial vision of the proving ground it will have all the standard endurance testing features like a "conventional" proving ground, but all the tracks are designed in a way that they incorporate all the necessary requirement to be able to test the vehicles' connected and automated features.

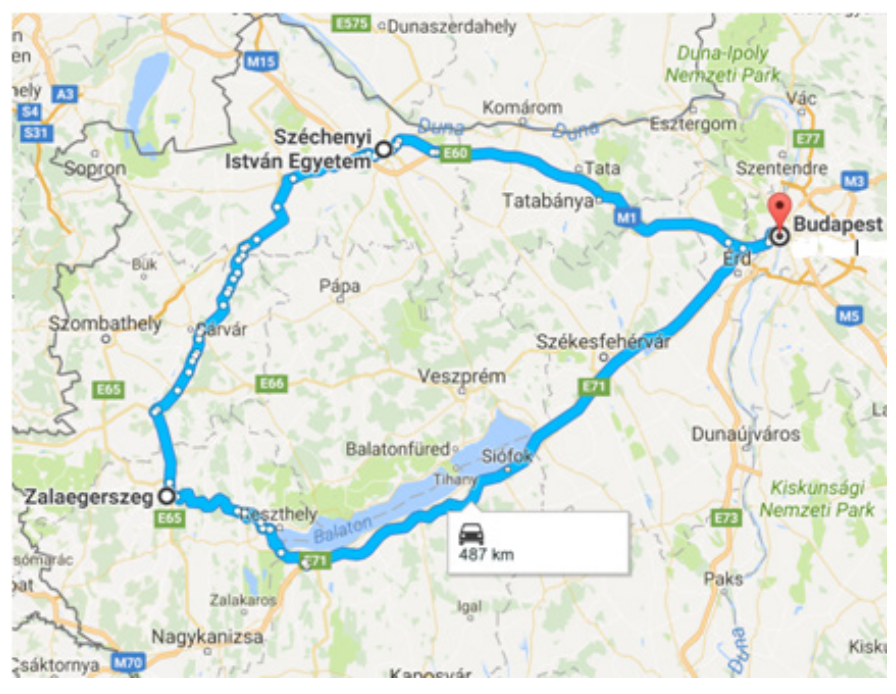

Fig. 6 Roads in Hungary where public road tests will be allowed

The elements of the proving ground are defined by the pyramid of the testing and validation of connected and automated vehicles (see Fig. 4).

In the proving ground design there are workplaces to do simulation work, and there are also buildings dedicated to special laboratories. The test track takes place on the largest area of the facility. Limited public road tests could be performed in a pre-chosen part of Zalaegerszeg, this area would have "Smart City" features.

Public road tests could be performed on the highways between Budapest and Zalaegerszeg, this is going to be regulated in a code modification in preparation by the government. The selected road sections were presented in Fig. 6.

The test track of Zalaegerszeg will also able to serve for standard vehicle dynamics testing, but it focuses on fully integrated autonomous vehicle testing and validation.

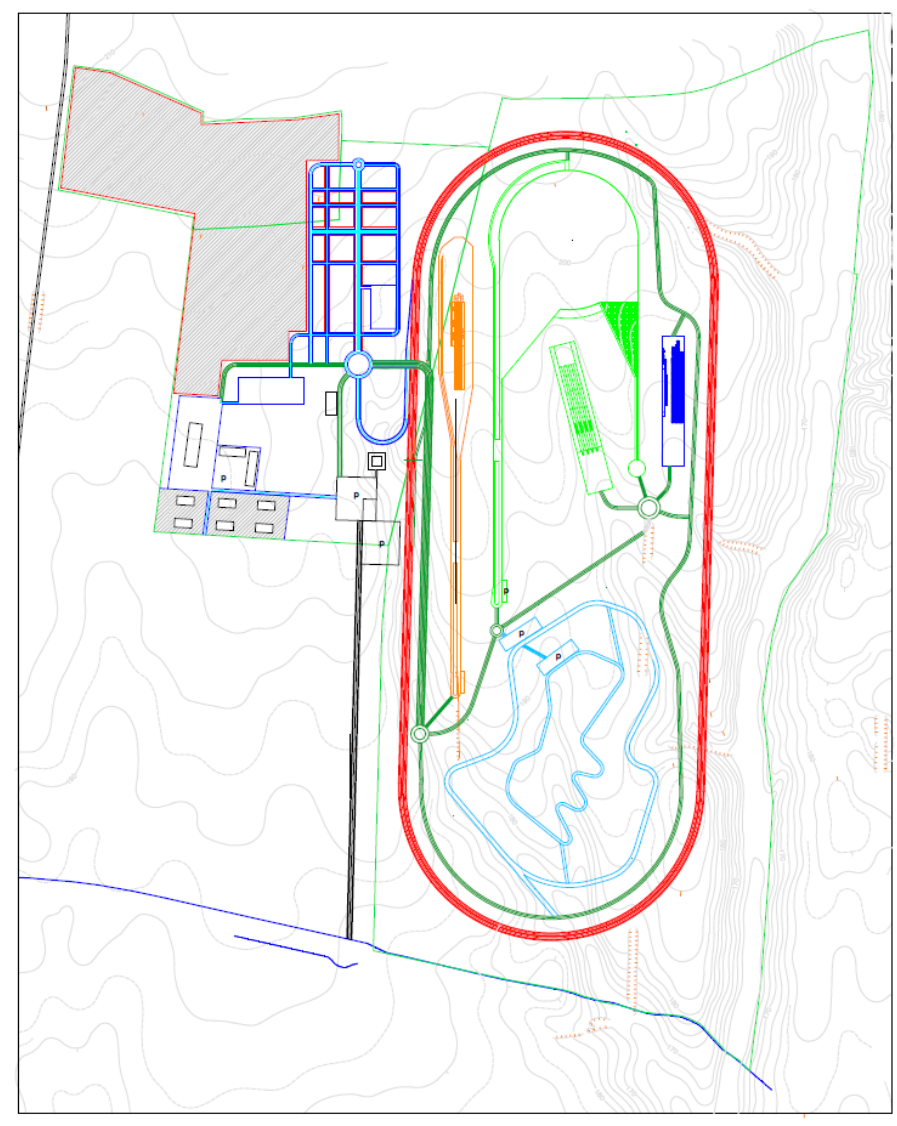

Fig. 7 Automotive Proving Ground dedicated to autonomous vehicle testing and validation at Zalaegerszeg, test track design

The test track design has the following parts:

- Urban tracks for autonomous vehicles: this part has complex city road elements, like traffic crossings with traffic signs, streets, parking places and buildings, and it has special equipments (for example moving obstacles) too. Smart City features (like intelligent lighting) are also going to be realized here.

- Rural roads: to test the vehicles in lower velocities. The shape of this road is designed to be a challenge for autonomous systems.

- General highway road: to test high speed autonomous functions like lane keeping or changing.

- Other alternative grounds to test the vehicles on roads with different qualities, slopes, or to do measurements.

- Dedicated grounds to test C2X communication technologies. 
The workshops of the working groups were scheduled monthly. Every participant presented their general and specific expectations about the proving ground. The interests of automotive organisation units were different, some of them expected conventional vehicle test possibilities, and others expected automated and connected vehicle testing features. The specification will contain both requirements.

The working group and the tasks were divided to a few subgroups. Based on the task of the sub-groups the participants decided which ones fit into their competence, and they worked in them. In the regular workshops the participants shared their experiments and opinions, and after the iteration of the discussions, the working group made an agreement: an internal approval for the specification.

This level of cooperation between the industrial companies, the universities and research centres and the national authorities guarantees that the proving ground will be beneficial for all of the automotive organisation units in Europe.

\section{Conclusion}

In the next ten years automated and connected vehicles are going to radically change road transport. The major motivation for their spreadding is to improve road safety by removing human error from the driving equation. But how can we guarantee that the operation of connected and automated vehicles is safer than human driven conventional vehicles? This paper tries to present a basic research results as answer to this question.

The appearance of connected and automated vehicles is a major technological step. Improving the safety of connected and automated vehicles, the key is defining the testing and validation processes for the new autonomous systems and features. These new testing and validation processes have a great development potential.

In this paper a new approach is worked out to structure the testing and validation processes. We have classified the processes by the V-model of system development and by the testing environment. Apart from the proof of safety it is necessary to develop a cost and time efficient methodology.

In the framework of the RECAR program we have deployed the plan for the testing and validation of connected and automated vehicles. This is classified by the layers of the connected and automated vehicle testing and validation "pyramid". The methodology guarantees that our specification contains the experiments of the automotive industrial and research centre's partners. The specification also contains the plan all of the "pyramid's" layers, but it focuses mainly on the 3rd one: the technical parameters of the test track in Zalaegerszeg.

We hope we will have the chance to validate our ideas and new testing and validation methodologies can be based on this paper. Apart from that we also hope that the new test track will serve the competitiveness of the region.

\section{References}

Aradi, Sz., Bécsi, T., Gáspár, P. (2014). Experimental Vehicle Development for Testing Autonomous Vehicle Functions. In: 2014 IEEE/ASME 10th International Conference on Mechatronic and Embedded Systems and Applications (MESA). Senigallia, Ancona, Italy, Sep. 10-12, 2014, pp. 1-5. https://doi.org/10.1109/MESA.2014.6935534

D2.1 - Accident Analysis, Naturalistic Observations and Project Implications (Wisch et al., 2016)

D2.2 - Specification of User Needs (drivers and VRUs) and Functional Requirements of Vehicle Safety Systems (Large et al., 2016)

D8.2 - Policy Support Strategy (Bartels et al., 2016)

Gáspár, P., Szalay, Zs., Aradi, Sz. (2014). Highly Automated Vehicle Systems. BME MOGI. URL: http://www.mogi.bme.hu/TAMOP/jarmurendszerek_iranyitasa_angol/index.html

International Organization for Standardization. (2011). International standard road vehicles - functional safety. International Organization for Standardization, Geneva, Switzerland. ISO 26262-1:2011.

Kalra, N., Paddock, S., M., (2016). Driving to safety: How many miles of driving would it take to demonstrate autonomous vehicle reliability? RAND Corporation. URL: http://www.rand.org/content/dam/rand/pubs/ research_reports/RR1400/RR1478/RAND_RR1478.pdf

Kato, S., Takeuchi, E., Ishiguro, Y., Ninomiya, Y., Takeda, K., Hamada, T. (2015). An Open Approach to Autonomous Vehicles. IEEE Micro. 35(6), pp. 60-68. https://doi.org/10.1109/MM.2015.133

Li, L., Huang, W. L., Liu, Y., Zheng, N. N., Wang, F. Y. (2016). Intelligence Testing for Autonomous Vehicles: A New Approach, IEEE Transactions on Intelligent Vehicles. IEEE Transactions on Intelligent Vehicles. 1(2), pp. 158-166.

https://doi.org/10.1109/TIV.2016.2608003

SAE International (2014). Taxonomy and Definitions for Terms Related to On-Road Motor Vehicle Automated Driving Systems, SAE standard, nr. J3016_201401,2014-01-16. URL: http://standards.sae.org/j3016_201401/

Szalay, Zs. (2016). Structure and Architecture Problems of Autonomous Road Vehicle Testing and Validation. In: 15th Mini Conference on Vehicle System Dynamics, Identification and Anomalies - VSDIA. BME, Budapest, Hungary, Nov. 7-9, 2016.

Tettamanti, T., Varga, I., Szalay, Zs. (2016). Impacts of Autonomous Cars from a Traffic Engineering Perspective. Periodica Polytechnica Transportation Engineering. 44(4), pp. 244-250. https://doi.org/10.3311/PPtr.9464

Winston, C., (2016). Can markets address government failure? The case of autonomous vehicles. In: Transforming transportation with autonomous vehicles and the sharing economy: Are we ready? Brookings Institution, Washington, DC. 